\title{
Original
}

\section{Construction and Expression of Ryanodine Receptor Mutants Relevant to Malignant Hyperthermia Patients in Japan}

\author{
Masahide NAKANO ${ }^{1,2)}$, Hideto OYAMAdA ${ }^{1)}$, Toshiko YamazawA ${ }^{3)}$, \\ Takashi MuraYAmA ${ }^{4)}$, Hokuto NANBA ${ }^{1)}$, Kentaro IJjIMA ${ }^{1)}$ \\ and Katsuji OGUCHI ${ }^{1)}$
}

\begin{abstract}
Malignant hyperthermia ( $\mathrm{MH})$ is a potentially fatal pharmacogenetic disorder triggered by exposure to commonly used volatile anesthetics. Pharmacological and genetic analyses implicated the type 1 of ryanodine receptor (RyR1)/ $\mathrm{Ca}^{2+}$ release channel as the main candidate gene for causing $\mathrm{MH}$. Genetic diagnosis of $\mathrm{MH}$ was proposed to replace conventional methods using biopsied muscle samples that are painful for patients and require skillful diagnosticians to interpret. However, more than 250 RyR1 gene variants have now been reported in MH-susceptible patients, although most have yet to be associated with functional abnormalities using exogenous constructs of these mutants expressed in living cells. To directly compare the pharmacological characteristics of some of the MH-related RyR1 mutants, we have established doxycycline -inducible cell lines expressing two of the unconfirmed rabbit RyR1 mutants, Q156K or R534H (corresponding to the Q155K or $\mathrm{R} 533 \mathrm{H}$ mutations in human RyR1 reported in $\mathrm{MH}$ patients in Japan) and a confirmed mutant, R164C RyR1 (corresponding to the R163C mutation in human). The caffeine sensitivity of Q156K-expressing cells was remarkably enhanced compared to wild-type RyR1 and similarly to previously reported levels for R164C-expressing cells, while that of the R534H mutants was not different from wild-type cells. The resting cytosolic $\mathrm{Ca}^{2+}$ concentrations of cell lines expressing Q156K or R164C were much higher than those expressing R534H or wild-type RyR1. These results indicated that the RyR1 gene mutation causing the Q156K phenotype (Q155K in human) is potentially susceptible to $\mathrm{MH}$, and that screening for this mutation could be useful for the noninvasive genetic diagnosis of $\mathrm{MH}$ in humans.
\end{abstract}

Key words : malignant hyperthermia, genetic diagnosis, ryanodine receptor, $\mathrm{Ca}^{2+}$ release channel

\footnotetext{
1) Department of Pharmacology, Showa University School of Medicine, 1-5-8 Hatanodai, Shinagawa-ku, Tokyo 142-8555, Japan.

2) Department of Surgery, Showa University Northern Yokohama Hospital.

3) Department of Molecular Physiology, Jikei Medical University School of Medicine.

4) Department of Pharmacology, Juntendo University School of Medicine.
} 


\section{Introduction}

Malignant hyperthermia $(\mathrm{MH})$ is a potentially fatal pharmacogenetic disorder triggered by exposure to commonly used volatile anesthetics. $\mathrm{MH}$ has severe clinical symptoms including rapid increases in body temperature due to accelerated muscle metabolism, muscle contractures resulting in metabolic acidosis, tachycardia, and rhabdomyolysis ${ }^{1,2)}$. If not immediately treated, these symptoms are likely to be fatal. MH susceptibility is difficult to predict in patients prior to anesthesia because susceptible individuals are clinically normal in the absence of triggering agents. The main accepted and validated tests for detecting $\mathrm{MH}$-susceptible individuals or families are the in vitro contracture test (IVCT) recommended by the European Malignant Hyperthermia Group (EMHG) ${ }^{3)}$ and the caffeine-halothane contracture test (CHCT) recommended by the North American Malignant Hyperthermia Group ${ }^{4}$. Both tests are based on the muscle contracture tensions induced by caffeine and/or halothane in skeletal muscle biopsy specimens. Such biopsies are painful for patients and the diagnosis of $\mathrm{MH}$ susceptibility requires significant clinical skill, making easy and noninvasive or minimally invasive diagnostic procedures such as genetic analysis of blood samples desirable ${ }^{5,6)}$.

Biochemical and physiological studies using human $\mathrm{MH}$ patients and porcine $\mathrm{MH}$ models showed an abnormal calcium release from the sarcoplasmic reticulum (SR) in skeletal muscle ${ }^{7,8)}$. Furthermore Endo et $a l^{9)}$ and Kawana et $a l^{10)}$ demonstrated that the $\mathrm{Ca}^{2+}$ sensitivity and the maximum rate of $\mathrm{Ca}^{2+}$-induced $\mathrm{Ca}^{2+}$ release (CICR) in the muscle cells of $\mathrm{MH}$ patients were much higher than those in control muscle. The $\mathrm{Ca}^{2+}$ release channel protein responsible for CICR was isolated through its high affinity binding to the plant alkaloid "ryanodine"; hence it was named the ryanodine receptor $(\mathrm{RyR})^{11)}$. Molecular cloning of the RyR gene revealed three types of RyR isoforms (types 1, 2, and 3) in mammalian chromosomes, with type 1 of RyR (RyR1) dominantly expressed in skeletal muscle cells ${ }^{12}$. The RyR1 gene is one of the largest and most complex gene identified, with 106 exons and a transcript of over 15 kilo base-pairs encoding approximately 5000 amino acids in humans ${ }^{13)}$.

Mutation screening in the $\mathrm{MH}$ pig models at first revealed a nucleotide replacement of cytosine (C) at 1843 in the coding region of the RyR1 gene with thymidine (T), causing a point mutation of the ${ }^{615}$ arginine $(\mathrm{R})$ to cysteine $(\mathrm{C})(\mathrm{R} 615 \mathrm{C})$ in the primary structure ${ }^{14)}$. The R615C mutation has been identified in all $\mathrm{MH}$-susceptible strains, indicating the high degree of selective inbreeding in commercially reared pig.

In contrast, more than 250 mutation sites have been reported in the RyR1 genes of MH-susceptible humans ${ }^{15,16)}$, and interest is now focused on the functional effects of these variants for both understanding the molecular basis of $\mathrm{MH}$ and increasing the repertoire of mutations that could be useful diagnostically. According to the EMHG guidelines (http://www. emhg.org/genetics/mutations-in-ryr1/), only 31 variants of diagnostic value have been functionally characterized, and if not detected the physiological IVCT of an excised muscle biopsy specimen should be used.

In this study, we constructed two functionally uncharacterized RyR1 mutations, Q156K and 
$\mathrm{R} 534 \mathrm{H}$, in the rabbit RyR1 gene [corresponding to Q155K and R533H in humans, as identified in $\mathrm{MH}$ patients in Japan ${ }^{17)}$ ] and analyzed the recombinant RyR1s expressed in cultured cells by directly monitoring caffeine-induced intracellular $\mathrm{Ca}^{2+}$ release. Importantly, we found that one of these mutants, Q156K, conferred much higher sensitivity to caffeine than wild-type (WT) RyR1 as a normal control, while cells expressing another mutant, R534H, showed the same caffeine sensitivity as WT RyR1-expressing control cells. Therefore, genetic screening of patient blood samples for expression of the RyR1 Q155K mutant could be useful to predict MH disease susceptibility.

\section{Materials and Methods}

\section{Oligonucleotide-directed mutagenesis of MH-associated RyR1 mutants}

Three kinds of site-directed mutagenesis were induced to substitute the glutamine or arginine at position 164 or 534, respectively, with lysine (Q156K), the cysteine (R164C) in the cDNA cassette 1 (C1) (Xba I/Sal I) or the histidine (R534H) in cassette 2 (C2) (Sal I/Bsu 36I), which were excised from the full-length sequence in pBluescript KS $(+)$ (pBS-RYR1) ${ }^{18)}$. Three primer pairs were used for PCR amplifications of the each cDNA cassette template in the pBS2 vector as described below. For Q156K, Q156K-F (forward primer) (5'-AAGAAGAGATCTGA AGGAGAGAAGGTCCGC-3') and Q156K-R (reverse primer) (5'-TTCAGATCTCTTCTTGGA GGCTGGGTGCAT-3') ; for R164C, R164C-F (5'-GAAGGTCTGCGTCGGGGACGACCTCA-3') and R164C-R (5'-CCGACGCAGACCTTCTCTCCTTCCGA-3') or for R534H, R534H-F (5' -GGGGCAATCATGCCAATTGTGCCCTCTTCTCCAC-3') and R534H-R (5'-GAGGGCACA ATTGGCATGATTGCCCCGGATCAGAG-3'). DNA sequencing was undertaken to validate the nucleotide sequence in each mutated cDNA cassette, with reactions performed in a BioRad DNA Engine Dyad PTC-220 Peltier Thermal Cycler (Bio-Rad Laboratories, Inc., Hercules, CA, USA) using an ABI BigDye ${ }^{\mathrm{TM}}$ Terminator v3.1 Cycle Sequencing Kit with AmpliTaq DNA polymerase (Applied Biosystems, Inc., Foster City, CA, USA), following the protocols supplied by the manufacturer. The sequencing reactions were then subjected to electrophoresis in an ABI 3730xl sequencer (Applied Biosystems, Inc.).

Each confirmed cDNA cassette was inserted back into the same position in pBS-RYR ${ }^{18)}$ to yield the full-length cDNA encoding Q156K, R164C, or R534H RyR1.

\section{Establishment of stable cell lines expressing doxycycline-inducible RyR1 mutants}

The Flp-In ${ }^{\mathrm{TM}}$ T-REx ${ }^{\mathrm{TM}}$ Systems (Invitrogen, Carlsbad, CA, USA) was also employed as described by Hayashi et $a l^{19)}$, but with additional refinement procedures to establish the stable cell lines expressing full-length RyR1. In brief, the Flp-In TREx293 cells (Invitrgen) were cultured in Dulbecco's modified Eagle's medium (Wako Pure Chemicals Industries, Ltd., Osaka, Japan) with $10 \%$ fetal bovine serum and 100 units of penicillin $/ \mathrm{mL}, 100 \mu \mathrm{g}$ of streptomycin/mL (Wako Pure Chemicals Industries, Ltd.). The tetracycline-inducible expression vector pcDNA5/ FRT/TO (Invitrogen) was modified by the addition of an NheI site in its multicloning sites to insert the full-length RyR1 cDNA digested by XbaI/EcoRV between the NheI and EcoRV 
sites. Co-transfection with the expression vector containing the full-length RyR1cDNA and the pOG44 vector encoding Flp recombinase (Invitrogen) was carried out by the calcium phosphate precipitation method of the Profection Mammalian Transfection System Kit (Promega Corp., Madison, WI, USA). Transfected cells were washed four hours after the transfection and allowed to grow in fresh medium for two days, and then the growth medium was replaced with the same medium containing $200 \mu \mathrm{g} / \mathrm{mL}$ hygromycin B (Wako Pure Chemicals Industries) to select the transformed cells. The selection medium was changed every 3-4 days until the desired number of cells per colony was reached. Doxycycline, a stable tetracycline derivative, (Dox; $1 \mu \mathrm{g} / \mathrm{mL}$, Sigma-Aldrich Co., St. Louis, MO, USA) had been applied to the selected cells for 48-72 hours to induce the expression of full-length RyR1 proteins.

\section{Immunocytochemistry}

Stable cell lines of the transformed Flp-In TREx293 cells were washed with PBS, fixed with $4 \%$ formaldehyde, and permeabilized with $0.2 \%$ saponin (Wako Pure Chemicals Industries, Ltd.). After blocking with 4\% skim milk in phosphate-buffered saline (PBS), exogenously expressed RyR1 was detected with primary antibodies against the D2 region of RyR1 ${ }^{20)}$ and Alexa Fluor 546-conjugated anti-rabbit IgG antibody as the secondary antibody (Molecular Probes, Eugene, OR, USA). Subcellular localizations of RyRs were visualized under an inverted fluorescent microscope Eclipse-Ti-U (Nikon, Tokyo, Japan) equipped with a cooled charge-coupled device camera DS-2MBWc (Nikon).

\section{SDS-polyacrylamide gel electrophoresis (PAGE) and Western blotting}

SDS-PAGE was performed on $2-12 \%$ linear gradient gels with standards 205 (in $\mathrm{kDa}$ ) (myosin heavy chain), 116 (b-galactosidase), 97.4 (phosphorylase b) 66 (bovine serum albumin), and 45 (ovalbumin). Gels were stained with Coomassie Brilliant Blue and the separated proteins were electrophoretically transferred overnight onto PVDF membranes at 40 $\mathrm{V}$ in the presence $0.02 \%$ SDS. Immunodetection was carried out with an ECL system (GE HealthcareUK, Ltd. Little Chalfont, Buckinghamshire, England) using primary antibodies against RyR1 as previously described and peroxidase-conjugated secondary antibodies.

\section{Imaging of intracellular $\mathrm{Ca}^{2+}$ concentration}

Changes in intracellular $\mathrm{Ca}^{2+}$ levels of cultured cells were measured using the $\mathrm{Ca}^{2+}$ indicator dye Fura-2. Cells were loaded with $4 \mu \mathrm{M}$ Fura-2/AM (Molecular Probes) in a physiological salt solution (PSS; $150 \mathrm{mM} \mathrm{NaCl}, 4 \mathrm{mM} \mathrm{KCl}, 2 \mathrm{mM} \mathrm{CaCl}, 1 \mathrm{mM} \mathrm{MgCl} 2,5.6 \mathrm{mM}$ glucose, $5 \mathrm{mM}$ HEPES, adjusted to $\mathrm{pH} 7.4$ with $\mathrm{NaOH}$ ) containing $0.1 \%$ bovine serum albumin V (SigmaAldrich) for 30 minutes at room temperature. Cells were viewed under an inverted fluorescence microscope (IX70, Olympus Corp., Tokyo, Japan) equipped with a charge-coupled device camera (Rolera, QImaging, Burnaby, BC, Canada). Changes in the intracellular $\mathrm{Ca}^{2+}$ concentrations were measured by monitoring the ratio of fluorescence signals at $515-550 \mathrm{~nm}$ with the alternate excitation at 345 and $380 \mathrm{~nm}$. Image analysis was performed using IPLab software (Signal 
Analytics, Glen Allen, VA, USA) and images were converted to 345/380 ratio images by ImageJ software (National Institutes of Health, Bethesda, MD, USA) and then averaged over the whole area sampled. Images were captured by a high-sensitivity, charge-coupled device digital camera (DP70, Olympus Corp., Tokyo, Japan) using image-capture software (DP Control, Olympus Corp., Tokyo, Japan). Captured images were analyzed using ImageJ. The change in the ratio of fluorescence intensity at $345 \mathrm{~nm}$ to that at $380 \mathrm{~nm}$ was normalized by the averaged resting value before the first drug application.

\section{Results}

Mutagenesis for making cDNAs of the RyR1 cDNA mutant

Constructions of mutant cDNA cassette of RyR1 were performed by the oligonucleotidedirected mutagenesis method (see "Materials and Methods"), and nucleotide sequences were confirmed by restriction fragment length polymorphism (RFLP; data not shown) (see "Materials and Methods"; the gain of Bgl II site for Q156K, the loss of Ava II site for R164C or the gain of Mfe I site for R534H) and also by nucleotide sequencing within each RyR1 cDNA cassette (Fig. 1 A, B and C). This step was conducted to check that the exogenously expressed RyR1 proteins encoded by these mutants of RyR1cDNA have the replaced amino acid sequences of RyR1 as clinically reported for MH patients in Japan previously ${ }^{17)}$.

\section{Expression of the mutant RyR1 cDNAs}

The WT and mutant RyR1 constructs were expressed in TREx293 cells by the tetracyclineinducible expression system (see "Materials and Methods"). Fig. 2A shows Western blotting of the lysate from these cell lines. The anti-RyR antibodies detected protein bands for a high molecular-mass species $(565 \mathrm{kDa})$ in all the cells. No positive bands were observed in nontransfected cells (data not shown). The intensities of the RyR1 bands were similar among them, indicating that the levels of these recombinant protein expressions were similar. Fig. $2 \mathrm{~B}$ shows the localization of the mutant RyR1s in TREx293 cells detected by staining with anti- RyR1 antibodies and visualization with fluorescence labeled anti-rabbit IgG secondary antibody. As observed for the WT, all of these mutants exhibited network-like patterns in the cytoplasmic region rather than in the nucleus, indicating endoplasmic reticulum localization of the expressed RyR1. Consistent with the Western blots, the fluorescence intensities of the corresponding cultured cells were almost the same, also indicating similar levels of expression of these mutants to WT-RyR1.

\section{Caffeine-induced $\mathrm{Ca}^{2+}$ transients of TREx293 cells expressing RyR1s}

The mutant RyR1s were assessed by CICR activities. We initially determined the caffeineinduced $\mathrm{Ca}^{2+}$ transients in TREx293 cells. Caffeine is a potent activator of CICR and caffeineinduced $\mathrm{Ca}^{2+}$ transients well reflect such activity in vivo ${ }^{21)}$. There was no or only small increases in fluorescent $\mathrm{Ca}^{2+}$ indicator, fura2, intensities from non-transfected cells, indicating a negligible level of endogenous RyRs (data not shown). All kinds of mutant RyR1 clones 

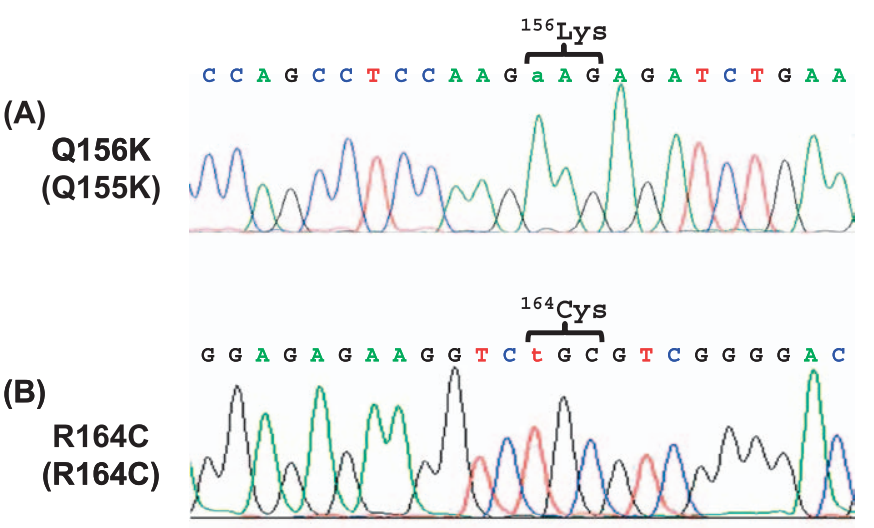

(C)

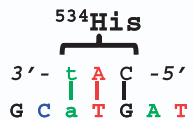

G C A C A A T T G G C a T G A T T G C C C C G

R534H (R533H)

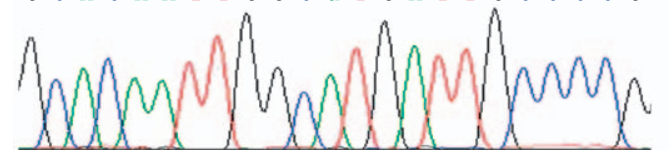

Fig. 1. DNA sequence confirmation of mutated coding sequences.

The chart records show the coding nucleotide sequences for Q156K (A), R164C (B), and the complimentary sequence for R534H (C). Each replaced nucleotide is written in lower case, whereupon its encoding substituted amino acid is indicated.

(A)

$565 \mathrm{kD}$

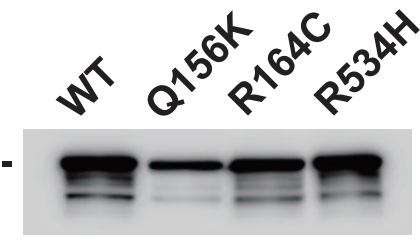

(B)

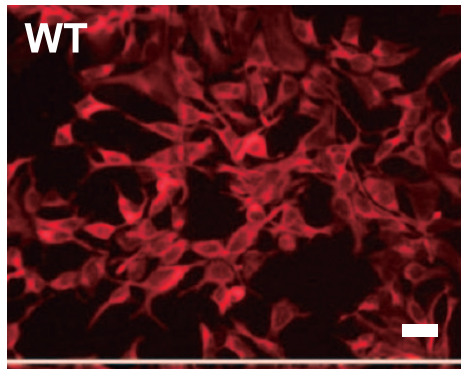

\section{Q156K}
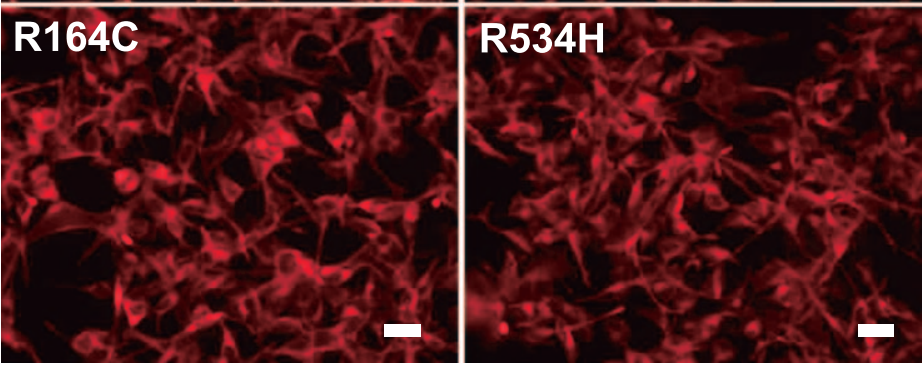

Fig. 2. Expression and localization of the RyR1mutants in cultured cell lines. Western blotting of RyR1 in the lysate of cell lines expressing WT or MH mutant RyR1s (A). The mutant RyR1s showed gel mobility similar to that of the WT gene $(565 \mathrm{kDa})$, and the expression levels of all mutants were nearly equivalent to that of the WT. Immunohistochemical localization of RyR1 in cultured cell lines (B). The WT and all RyR1 mutations gave rise to similar patterns in the cytosolic regions, but not in the nucleus, indicating the endoplasmic reticulum-representative localization. Scale bars indicate $100 \mu \mathrm{m}$. 
expressing TREx293 cells exhibited caffeine-induced $\mathrm{Ca}^{2+}$ transients in a dose-dependent manner : the $\mathrm{Ca}^{2+}$ transients increased as the caffeine concentrations increased (Fig. 3B, C and D). This is consistent with previous papers describing HEK293 cells (the original cell line for TREx293 cells) expressing RyR1 (Fig. 3A, 18). However, the peak magnitude of $\mathrm{Ca}^{2+}$ transients varied within each clone and peak values in the Q156K and R164C mutants were much less than that in WT-RyR1: the rank order was R164C $<$ Q156K $<\mathrm{R} 534 \mathrm{H} \fallingdotseq \mathrm{WT}$ (Fig. 4A). To determine caffeine sensitivities, the respective peak magnitudes of $\mathrm{Ca}^{2+}$ transients were plotted against the caffeine concentration after correction for caffeine effect (Fig. 4B). The rank order of caffeine sensitivity was Q156K > R164C > R534H $\fallingdotseq$ WT (Fig. 4B).

\section{Comparison of resting $\mathrm{Ca}^{2+}$ concentrations in exogenous RyR1-expressing cells}

Pathophysiological features of RyR-related disease include abnormal $\mathrm{Ca}^{2+}$ leakage from the intracellular $\mathrm{Ca}^{2+}$ storage sites (endoplasmic reticulum or especially sarcoplsmic reticulum in striated muscle cells) resulted in elevated resting cytoplasmic $\mathrm{Ca}^{2+}$ concentrations without any stimulating drugs ${ }^{22,23)}$. Thus, we compared the resting $\mathrm{Ca}^{2+}$ concentrations among RyR1 mutants and WT (Fig. 5). The resting $\mathrm{Ca}^{2+}$ level was significantly higher for Q156K and R164C than
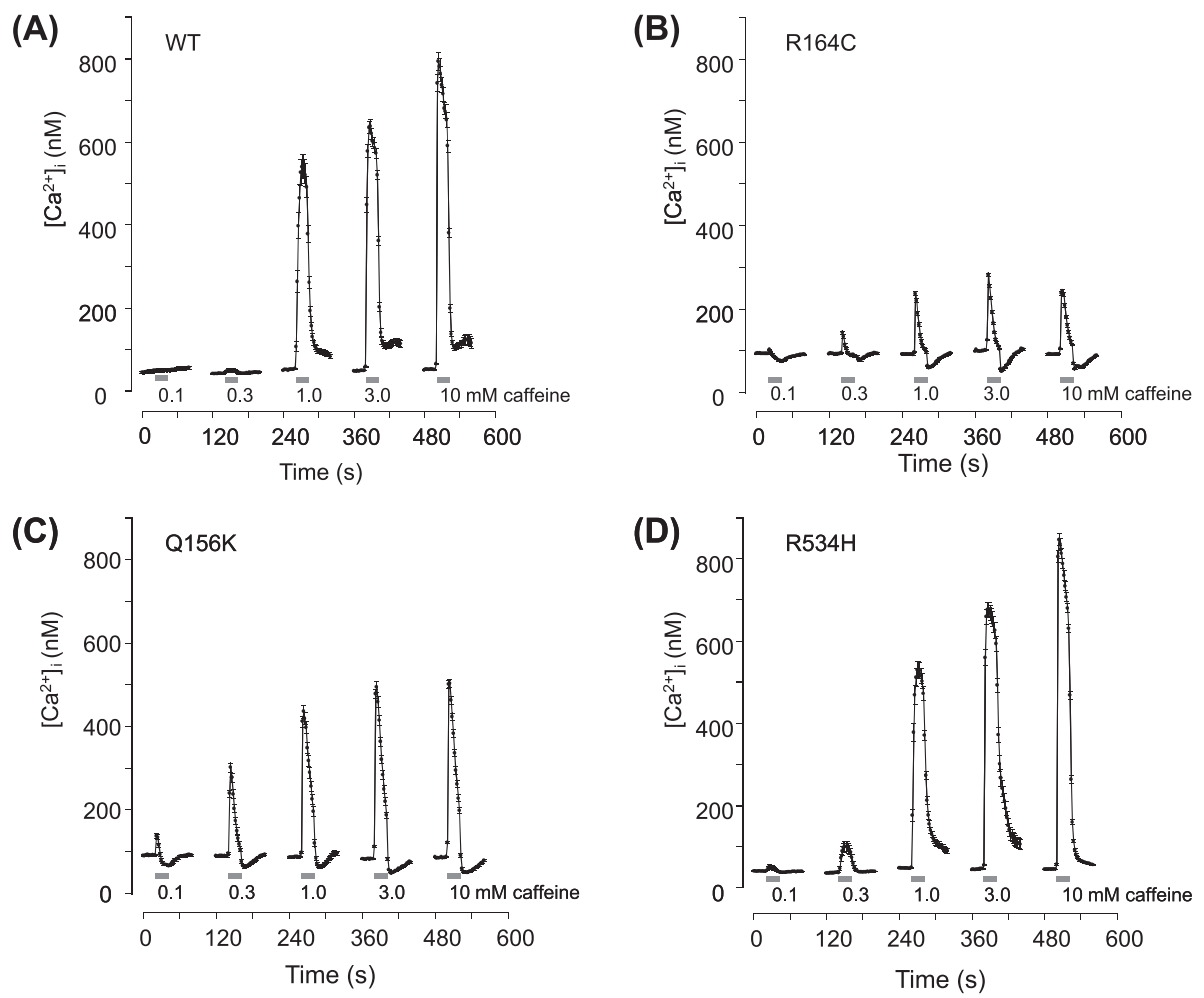

Fig. 3. Caffeine-induced $\mathrm{Ca}^{2+}$ release in cells expressing WT and mutant RyR1s. $\mathrm{Ca}^{2+}$ imaging traces from the fura-2-loaded living cells (see "materials and methods") expressing WT (A), R164C (B), Q156K (C), or R534H (D) mutations of RyR1. Caffeine was applied at the underlined times and the indicated concentrations. The dots in each trace represent mean \pm S.D. $(n=68-244$ cells in numbers $)$. 
(A)

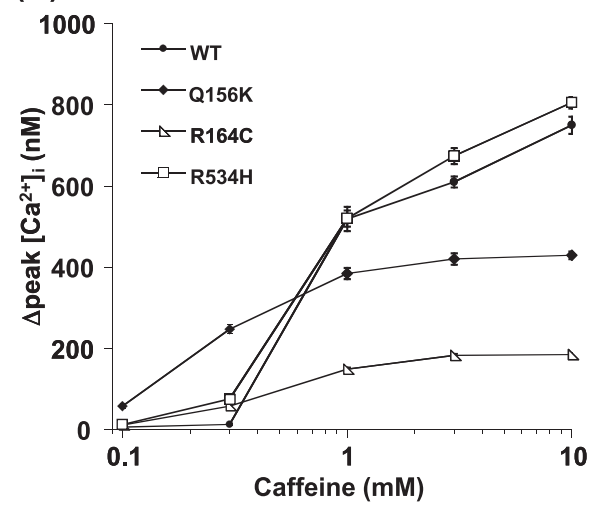

(B)

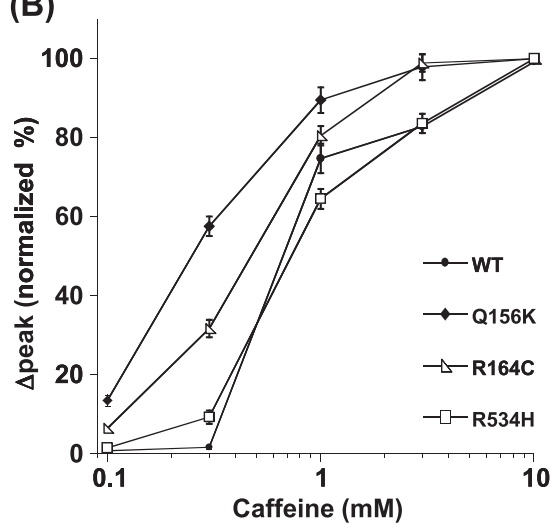

Fig. 4. Dose-response curves of the caffeine-induced $\mathrm{Ca}^{2+}$ release in cells expressing WT and mutant RyR1s.

The magnitudes of caffeine-induced $\mathrm{Ca}^{2+}$ concentration peaks different from under resting conditions in each cell line expressing exogenous RyR1 were plotted against the applied caffeine concentrations on horizontal lines (A). To compare the caffeine sensitivity of the mutated RyR1-expressing cells to that of WT, the initial peak amplitudes at each caffeine concentration were normalized to the maximum amplitude of the peak response in the fura-2 fluorescent ratio caused by $10 \mathrm{mM}$ caffeine (B). All dotted data represent mean \pm S.D. calculated from the experimental data in Fig. 3.

for WT, while that for R534H was similar to that for WT. These findings suggested that RyR1 mutants (Q156K and R164C) constitute phenotypically as leaky channels expressed in the cultured cells under resting conditions ${ }^{24)}$.

\section{Discussion}

The aim of this study was to analyze the sensitivity of functionally uncharacterized mutations ( Q156K and $\mathrm{R} 534 \mathrm{H}$ of rabbit RyR1 corresponding to the $\mathrm{Q} 155 \mathrm{~K}$ and the $\mathrm{R} 533 \mathrm{H}$ of humans) found in $\mathrm{MH}$ patients in Japan ${ }^{17)}$ to caffeine, a specific RyR agonist. We succeeded in constructing full-length cDNAs of these RyR1 gene variants and established stable cell lines expressing the RyR1mutants by addition of doxcycline. We found a lower peak $\mathrm{Ca}^{2+}$ concentration in the Q156K mutant cells induced by the addition of caffeine than in the WT-RyR1 cells, but not in the R534H mutant (Fig. 4A), and resting $\mathrm{Ca}^{2+}$ concentrations in the Q164K-expressing cells were much higher than those in the WT cells or those expressing the R534H RyR1 (Fig. 5). These results are consistent with the proposal that the Q156K RyR1 might allow more leakage of $\mathrm{Ca}^{2+}$ from the intracellular $\mathrm{Ca}^{2+}$ stores than the WT or $\mathrm{R} 534 \mathrm{H}$ receptor $^{24)}$. After normalization of all data as percentages (Fig. 4B), the markedly enhanced caffeine sensitivity was confirmed in the mutant Q156K- expressing cells, as the reported R164C- expressing cells in several previous studies ${ }^{24-26)}$, although $\mathrm{R} 534 \mathrm{H}$ expression conferred no enhancement in caffeine sensitivity compared to WT RyR1.

Recently, Sato et $a l^{27)}$ showed enhanced drug sensitivity with transient expression of the $\mathrm{R} 533 \mathrm{H}$ mutant of human RyR1 (corresponding to the R534H of rabbit RyR1) in HEK 293 


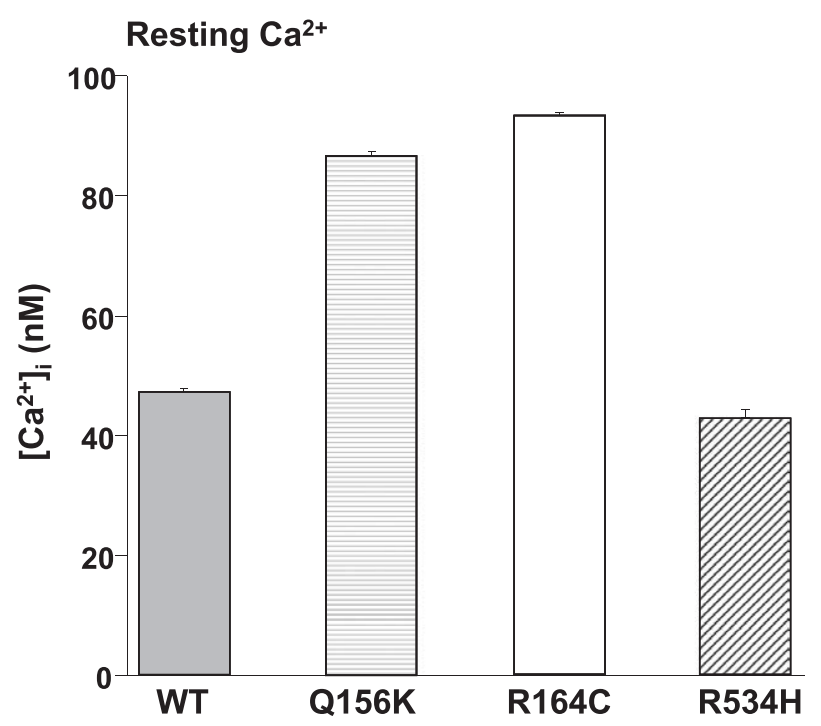

Fig. 5. Comparison of resting $\mathrm{Ca}^{2+}$ concentrations between WT and mutated RyR1-expressing cells.

Resting $\mathrm{Ca}^{2+}$ levels of cells expressing RyR1 mutants Q156K or R164C were higher than those in WT RyR1 cells, while those in the R534H-expressing cells were similar to WT levels. A similarly higher cytosolic $\mathrm{Ca}^{2+}$ concentration in $\mathrm{R} 164 \mathrm{C}$-expressing cells under resting condition was previously observed in different experimental systems ${ }^{24-26)}$.

cells using a plasmid vector containing the full-length mutated RyR1 cDNA of about 20,000 base pairs in length. In such an experiment, most cells were thought to express no or little recombinant proteins of RyR1 by the procedure for the transient expression since the efficiency of the gene transfer strongly depends on the nucleotide size of the expression vectors [see Fig. 2 in Sato et $\mathrm{al}^{27)}$. Even within the transfected cells, cell-to-cell variations in the expression levels of RyR1 would arise without selective pressure of antibiotic-resistance in the expression vectors. For these reasons, we used the established stable cell lines all of which could express the $\mathrm{MH}$ related RyR1 mutants at nearly the same level (Fig. 2B), resulting in high reproducibility for the expression level and the drug sensitivity of exogenous RyR1. In the paper of Sato et $a l^{27)}$, the human R163C RyR1 (corresponding to the rabbit R164C in this paper) produced the same resting $\mathrm{Ca}^{2+}$ concentrations as the human WT-RyR1, while the resting $\mathrm{Ca}^{2+}$ concentrations with the rabbit R164C or mice R163C RyR1 mutants studied in other papers ${ }^{24-26)}$ was much higher than with the WT-RyR1, and we showed the same result in the current study (Fig. 5). Sato and colleagues ${ }^{27)}$ used the drug "4-chloro-m-cresol $(4 \mathrm{CmC})$ ", which is different from the "caffeine" commonly used in the IVCT or CHCT protocols for the pre-symptomatic diagnosis of $\mathrm{MH}$ recommended by the EMHG or North American Malignant Hyperthermia Group, respectively,4). In a clinical paper on mutation screening, Ibarra et al ${ }^{17)}$ reported that MH-susceptible Japanese patients carrying the R533H mutation also had another RyR1 gene mutation encoding P1592L, and preliminary results with the P1593L mutant (corresponding 
to the P1592L in human RyR1) indicated enhanced drug sensitivity to caffeine [personal communication with Murayama T. et al (Dept. Pharmacol., Sch. Med., Juntendou Univ.)]. Therefore, the $\mathrm{R} 533 \mathrm{H}$ mutation in human RyR1 cannot be deemed a candidate for predicting $\mathrm{MH}$ susceptibility in patients.

The Q156K mutation in rabbit RyR1 is located in the amino $\left(\mathrm{NH}_{2-}: \mathrm{N}\right)$ terminal region of RyR1 termed the "MH/CCD (central core disease) region 1". This region is one of three distinct clusters in the linear sequence of RyR1, the amino-terminal (1-614 a.a.), central (21172458 a.a.), and carboxyl-terminal (4136-4973 a.a.) regions of RyR1, corresponding to MH/CCD regions 1,2 , and 3, respectively ${ }^{28)}$. Recently the $\mathrm{N}$-terminal region of RyR1 was crystallized and the subsequently solved structure revealed the disease-associated mutation "hot spot" loop (HS loop) in domain A, one of the three domains A (1-206 a.a.), B (207-394 a.a.), and C $(395-559 \text { a.a. })^{29,30)}$. The $\mathrm{Q} 156 \mathrm{~K}$ mutation is proposed to lie at the surface of the $\beta$-trefoil structure in the "HS loop" of domain A, wherein the uncharged side chain of glutamine (Q) must be changed to the positively charged lysine $(\mathrm{K})$. This conversion of a charged residue may perturb the quaternary RyR1 interactions involved in tetrameric channel formation or interactions with modulatory proteins. Solving the crystal structure of the expressed recombinant Q156K mutant might therefore provide a framework by which to understand the MH diseaseassociated mutations in RyR1 that have been studied using functional methods in this paper. Such molecular information will also be useful for developing new strategies to modulate RyR1 function in disease states.

Ongoing investigations into the functional expression of MH-related RyR1 mutants will uncover further causative mutations and thereby improve the usefulness and efficacy of diagnostic genetic testing for $\mathrm{MH}$ in the future.

\section{Acknowledgements}

We thank Mr. Atsuki Ogata and Miss Hiromi Shiiba for technical assistance.

\section{Conflict of interest}

The authors in this paper have no conflict of interest to disclose with respect to this publication.

\section{References}

1) Denborough MA, Lovell RRH. Anaesthetic deaths in a family. Lancet. 1960;2:45.

2) Hopkins PM. Malignant hyperthermia: pharmacology of triggering. Br J Anaesth. 2011;107:48-56.

3) A protocol for the investigation of malignant hyperpyrexia ( $\mathrm{MH}$ ) susceptibility. The European Malignant Hyperpyrexia Group. Br J Anaesth. 1984;56:1267-1269.

4) Larach MG. Standardization of the caffeine halothane muscle contracture test. North American Malignant Hyperthermia Group. Anesth Analg. 1989;69:511-515.

5) Girard T, Treves S, Voronkov E, et al. Molecular genetic testing for malignant hyperthermia susceptibility. Anesthesiology. 2004;100:1076-1080. 
6) Bandschapp O, Girard T. Malignant hyperthermia. Swiss Med Wkly. 2012;142:w13652.

7) Kalow W, Britt BA, Terreau ME, et al. Metabolic error of muscle metabolism after recovery from malignant hyperthermia. Lancet. 1970;2:895-898.

8) Ohta T, Endo M, Nakano T, et al. Ca-induced Ca release in malignant hyperthermia-susceptible pig skeletal muscle. Am J Physiol. 1989;256:C358-367.

9) Endo M, Yagi S, Ishizuka T. Changes of the Ca-induced Ca release mechanism in sarcoplsmic reticulum from a patient with malignant hyperthermia. Biomed Res. 1983;4:83-92.

10) Kawana $\mathrm{Y}$, Iino $\mathrm{M}$, Horiuchi $\mathrm{K}$, et al. Acceleration in the calcium-induced Ca release in the biopsied muscle fibers from patients with malignant hyperthermia. Biomed Res. 1992;13:287-297.

11) Inui M, Saito A, Fleischer S. Purification of the ryanodine receptor and identity with feet structures of junctional terminal cisternae of sarcoplasmic reticulum from fast skeletal muscle. J Biol Chem. 1987;262:1740-1747.

12) Takeshima H, Nishimura S, Matsumoto $T$, et al. Primary structure and expression from complementary DNA of skeletal muscle ryanodine receptor. Nature. 1989;339:439-445.

13) Phillips MS, Fujii J, Khanna VK, et al. The structural organization of the human skeletal muscle ryanodine receptor (RYR1) gene. Genomics. 1996;34:24-41.

14) Fujii J, Otsu K, Zorzato $\mathrm{F}$, et al. Identification of a mutation in porcine ryanodine receptor associated with malignant hyperthermia. Science. 1991;253:448-451.

15) Urwyler A, Deufel T, McCarthy $\mathrm{T}$, et al. Guidelines for molecular genetic detection of susceptibility to malignant hyperthermia. Br J Anaesth. 2001;86:283-287.

16) Stowell KM. Malignant hyperthermia: a pharmacogenetic disorder. Pharmacogenomics. 2008;9:1657-1672.

17) Ibarra M CA, Wu S, Murayama K, et al. Malignant hyperthermia in Japan: mutation screening of the entire ryanodine receptor type 1 gene coding region by direct sequencing. Anesthesiology. 2006;104:1146-1154.

18) Tong J, Oyamada H, Demaurex N, et al. Caffeine and halothane sensitivity of intracellular $\mathrm{Ca}^{2+}$ release is altered by 15 calcium release channel (ryanodine receptor) mutations associated with malignant hyperthermia and / or central core disease. J Biol Chem. 1997;272:26332-26339.

19) Hayashi $\mathrm{T}$, Oyamada $\mathrm{H}$, Yamazawa $\mathrm{T}$, et al. The role of a brain-specific splice variant of ryanodine receptor type 1. Showa Univ J Med Sci. 2010;22:105-115.

20) Murayama T, Oba T, Katayama E, et al. Further characterization of the type 3 ryanodine receptor (RyR3) purified from rabbit diaphragm. J Biol Chem. 1999;274:17297-17308.

21) Endo M. Conditions required for calcium-induced release of calcium from the sarcoplasmic reticulum. Proc Jpn Acad. 1975;51:467-472.

22) Lehnart SE, Wehrens XH, Laitinen PJ, et al. Sudden death in familial polymorphic ventricular tachycardia associated with calcium release channel (ryanodine receptor) leak. Circulation. 2004;109:3208-3214.

23) Bellinger AM, Reiken S, Dura M, et al. Remodeling of ryanodine receptor complex causes "leaky" channels: a molecular mechanism for decreased exercise capacity. Proc Natl Acad Sci U S A. 2008;105:2198-2202.

24) Tong J, McCarthy TV, MacLennan DH. Measurement of resting cytosolic $\mathrm{Ca}^{2+}$ concentrations and $\mathrm{Ca}^{2+}$ store size in HEK-293 cells transfected with malignant hyperthermia or central core disease mutant $\mathrm{Ca}^{2+}$ release channels. $J$ Biol Chem. 1999;274:693-702.

25) Yang T, Esteve E, Pessah IN, et al. Elevated resting $\left[\mathrm{Ca}^{(2+)}\right](\mathrm{i})$ in myotubes expressing malignant hyperthermia RyR1 cDNAs is partially restored by modulation of passive calcium leak from the SR. Am J Physiol Cell Physiol. 2007;292:C1591-C1598.

26) Giulivi C, Ross-Inta C, Omanska-Klusek A, et al. Basal bioenergetic abnormalities in skeletal muscle from ryanodine receptor malignant hyperthermia-susceptible R163C knock-in mice. J Biol Chem. 2011;286:99-113.

27) Sato K, Roesl C, Pollock N, et al. Skeletal muscle ryanodine receptor mutations associated with malignant hyperthermia showed enhanced intensity and sensitivity to triggering drugs when expressed in human embryonic kidney 
cells. Anesthesiology. 2013;119:111-118.

28) Robinson R, Carpenter D, Shaw MA, et al. Mutations in RYR1 in malignant hyperthermia and central core disease. Hum Mutat. 2006;27:977-989.

29) Amador FJ, Liu S, Ishiyama N, et al. Crystal structure of type I ryanodine receptor amino-terminal beta-trefoil domain reveals a disease-associated mutation "hot spot" loop. Proc Natl Acad Sci U S A. 2009;106:11040-11044.

30) Tung CC, Lobo PA, Kimlicka L, et al. The amino-terminal disease hotspot of ryanodine receptors forms a cytoplasmic vestibule. Nature. 2010;468:585-589.

[Received December 24, 2013 : Accepted January 8, 2014] 